\title{
Integrating HIV testing into immunological studies of non-HIV-related diseases
}

Siske S Struik, Caroline A Maxwell, Mwele Malecela-Lazaro, Sue Eckstein, John D H Porter \& Eleanor M Riley

Nature Immunology 6, 423-426 (2005).

The photo credit for Figure 2 on page 425 is incorrect. It should read "C. Drakeley" along the right edge and, in the legend, "Used with permission

from C. Drakeley." Nature Immunology regrets this error. 